\title{
Knockdown of POLA2 increases gemcitabine resistance in lung cancer cells
}

\author{
Vivien Koh ${ }^{1}$, Hsueh Yin Kwan ${ }^{2 \dagger}$, Woei Loon Tan ${ }^{1 \dagger}$, Tzia Liang Mah ${ }^{3 *}$ and Wei Peng Yong ${ }^{1,2^{*}}$ \\ From 15th International Conference On Bioinformatics (INCOB 2016) \\ Queenstown, Singapore. 21-23 September 2016
}

\begin{abstract}
Background: Gemcitabine is used as a standard drug treatment for non-small cell lung cancer (NSCLC), but treatment responses vary among patients. Our previous studies demonstrated that POLA2 + 1747 GG/GA single nucleotide polymorphism (SNP) improves differential survivability and mortality in NSCLC patients. Here, we determined the association between POLA2 and gemcitabine treatment in human lung cancer cells.

Results: Human PC9, H1299 and H1650 lung cancer cell lines were treated with 0.01-100 $\mu \mathrm{M}$ gemcitabine for $72 \mathrm{~h}$. Although all 3 cell lines showed decreased cell viability upon gemcitabine treatment, H1299 was found to be the most sensitive to gemcitabine treatment. Next, sequencing was performed to determine if POLA2 + 1747 SNP might be involved in gemcitabine sensitivity. Data revealed that all 3 cell lines harbored the wild-type POLA2 + 1747 GG SNP, indicating that the POLA2 + 1747 SNP might not be responsible for gemcitabine sensitivity in the cell lines studied. Silencing of POLA2 gene in H1299 was then carried out by siRNA transfection, followed by gemcitabine treatment to determine the effect of POLA2 knockdown on chemosensitivity to gemcitabine. Results showed that H1299 exhibited increased resistance to gemcitabine after POLA2 knockdown, suggesting that POLA2 does not act alone and may cooperate with other interacting partners to cause gemcitabine resistance.

Conclusions: Collectively, our findings showed that knockdown of POLA2 increases gemcitabine resistance in human lung cancer cells. We propose that POLA2 may play a role in gemcitabine sensitivity and can be used as a prognostic biomarker of patient outcome in NSCLC pathogenesis.
\end{abstract}

Keywords: POLA2, Gemcitabine, Non-small cell lung cancer, Drug response, Single nucleotide polymorphism

\section{Background}

Lung cancer is a leading cause of cancer mortality worldwide, accounting for approximately $28 \%$ of all cancer deaths annually. The prognosis for lung cancer is poor. Most lung cancer patients are diagnosed at advanced stage and only $16 \%$ remained surviving 5 years after initial diagnosis. Out of all lung cancer cases, about $85-90 \%$ of diagnosis are non-small cell lung cancer (NSCLC) [1]. NSCLC consists of three major tumor subtypes: adenocarcinoma, large-cell carcinoma and squamous-cell carcinoma. Therapeutic regimens are platinum-based doublets,

\footnotetext{
* Correspondence: tImah@i2r.a-star.edu.sg; Wei_Peng_YONG@nuhs.edu.sg ${ }^{\dagger}$ Equal contributors

${ }^{3}$ Institute for Infocomm Research, Agency for Science, Technology and Research, Singapore, Singapore

${ }^{1}$ National University Cancer Institute Singapore, National University Health System, Singapore, Singapore

Full list of author information is available at the end of the article
}

whereby the second agent can be microtubule-targeted agents, cytidine analogues or DNA-damaging agents [2]. Cytotoxic chemotherapy thus remains as the current standard cure for patients having advanced NSCLC.

Gemcitabine, a cytidine analogue, is well-known to have a significant therapeutic effect in NSCLC cases [3]. It is a prodrug that becomes activated by intracellular kinases to form di- and tri-phosphorylated metabolites, which together catalyze the conversion of ribonucleotides to deoxyribonucleotides and terminate DNA synthesis [4]. Studies have shown that the responses of NSCLC patients to gemcitabine treatment vary, which could be due to genetic polymorphisms and different gene variants involved in the gemcitabine pathway $[5,6]$.

The eukaryotic DNA polymerase $\alpha$, one of the main polymerases involved in nuclear DNA replication, is a four-subunit (A, B, C, D) enzyme which possesses DNA 
polymerase and primase activities. Earlier biochemical studies have reported that subunit A displays catalytic activity, while subunits $C$ and $D$ exhibit primase activity $[7,8]$. Subunit B, also known as DNA polymerase $\alpha 2$ accessory subunit (POLA2), is a $70-\mathrm{kDa}$ regulatory subunit which contributes to DNA replication by binding the DNA polymerase $\alpha$-primase complex to the initiation and elongation machinery [9]. POLA2 is widely expressed in a variety of tissue types. It has recently been shown to participate in gene fusion events and may also act as a prognostic biomarker in ovarian cancer and gastrointestinal stromal tumors $[10,11]$. However, the exact role of POLA2 in human cancer remains unknown and its involvement in NSCLC pathogenesis remains understudied.

We have previously demonstrated that POLA $2+1747$ GG/GA improves differential survivability and mortality in NSCLC patients, and proposed that this novel single nucleotide polymorphism (SNP) may be used as a prognostic biomarker of patient outcome in NSCLC pathogenesis [12]. In the current study, we sought to determine the association between POLA2 and gemcitabine treatment, and further characterized the role of POLA2 in human lung cancer cells.

\section{Results}

\section{Genomic landscape of POLA2}

To understand POLA2 genomic sequence, we first examined the sequence of POLA2 using the human Dec. 2013 (GRCh38/hg38) assembly on the UCSC Genome Browser (https://genome.ucsc.edu/). POLA2 is located on chromosome $11 \mathrm{q} 13.1$ and contains 21 exons. Assessment of the DNA sequence using data retrieved from the Encyclopedia of DNA Elements (ENCODE) indicated the presence of several integrated transcriptional regulatory elements. Comparative genomics analysis using multiple alignments of vertebrate species revealed conservation of the POLA2 gene. We further explored the presence of SNP sites within the POLA2 genomic region. Figure 1a shows the location of each SNP, as reported by the Single Nucleotide Polymorphism database (dbSNP) build 146. Only SNPs that have a minor allele frequency of at least $1 \%$ and are mapped to a single location in the reference genome assembly are included.

\section{Association of POLA2 with other genes}

Recent studies reported that POLA2 may participate in gene fusion events that can contribute to prognosis in ovarian cancer and gastrointestinal stromal tumors. Using data compiled from the National Center for Biotechnology Information (NCBI) and Gene Ontology (GO) framework databases, we uncovered 44 unique interactants for POLA2 (Table 1). These interactants were inferred from physical interactions, sequence or structural similarities, as well as direct experimental assays. Pathway analysis showed that POLA2 predominantly plays a major role in DNA replication, which involves a complex network of other interacting enzymes and proteins (Fig. 1b). Figure 1c further depicts the visualization of POLA2 and its interactants in a network, which was generated based on published and known physical or genetic interactions, chemical associations and post-translational modifications.

\section{Effect of gemcitabine on human lung cancer cell lines}

To examine the effect of gemcitabine on human lung cancer cell lines, we treated PC9, H1299 and H1650 cell lines with $0.01-100 \mu \mathrm{M}$ gemcitabine for $72 \mathrm{~h}$. As shown in Fig. 2, cell viability decreased with increasing concentrations of gemcitabine treatment. Although all 3 cell lines showed similar trends of decreasing cell viability upon gemcitabine treatment, H1299 was found to be the most sensitive to gemcitabine, having the lowest $\mathrm{IC}_{50}$ among the 3 cell lines studied.

\section{Detection of SNPs in human lung cancer cell lines}

The POLA + 1747 GG/GA SNP and SLC28A2 + 65 CC SNP are two of the 21 SNPs of the 9 genes involved in gemcitabine transport, metabolism and activity. From our in silico prediction analysis reported earlier [12], these two SNPs gave the best survival outcome and thus of interest for further studies in vitro. Here, sequencing of PC9, H1299 and H1650 cell lines revealed that all of these cell lines harboured the wild-type POLA2 + 1747 SNP (Fig. 3a), indicating that the POLA2 + 1747 SNP might not be responsible for gemcitabine sensitivity in the cell lines studied. Next, to investigate if SLC28A2 + 65 SNP might be involved in gemcitabine sensitivity, we sequenced PC9, H1299 and H1650 cell lines for the SLC28A2 + 65 SNP. As shown in Fig. 3b, only H1650 cell line harboured the mutation SLC28A2 +65 CC >CT SNP, whereas both PC9 and H1299 cell lines contained the wild-type SLC28A2 + 65 CC SNP. We therefore selected H1299 and H1650 cell lines for subsequent experiments.

\section{Knockdown of POLA2 gene increased resistance to gemcitabine treatment}

Both H1299 and H1650 cell lines were transfected for 48-72 $\mathrm{h}$ with the respective small interfering RNAs (siRNAs) to silence POLA2 and SLC28A2 genes. However, H1650 cell line could not be successfully transfected (data not shown), hence only the H1299 cell line was used for further studies. Western blot analysis showed that POLA2 protein expression was significantly attenuated after $72 \mathrm{~h}$ of transfection (Fig. 4a). A chemosensitivity assay was then conducted to determine the effect of POLA2 knockdown on the drug sensitivity of H1299 cell line to gemcitabine. As shown in Fig. 4b, H1299 cell line became more resistant to gemcitabine 


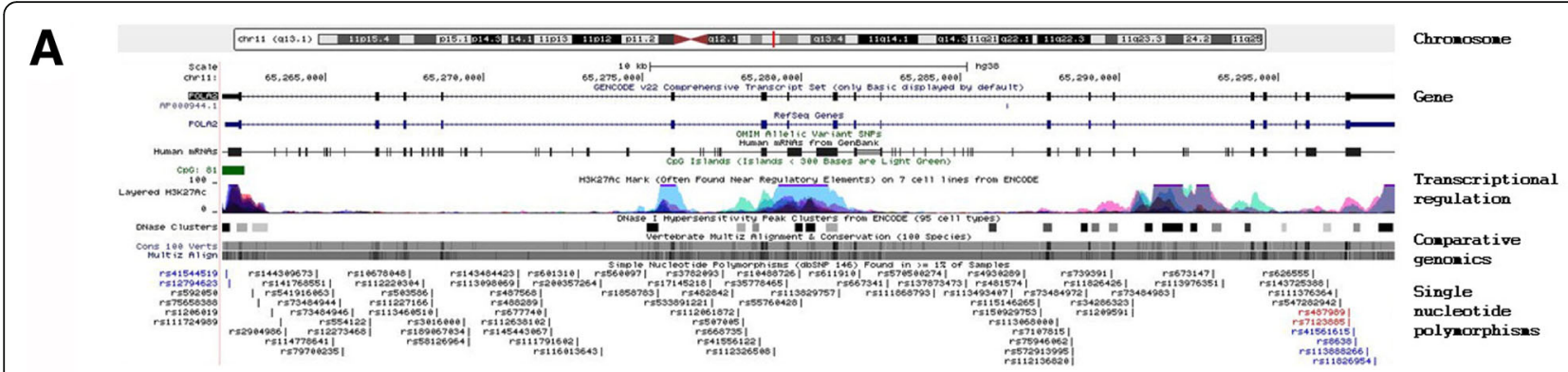

B

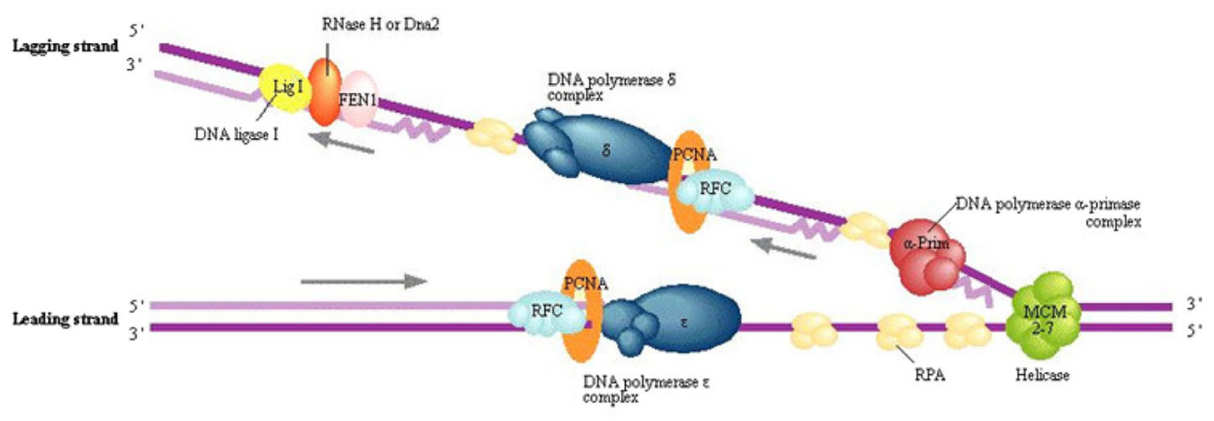

C

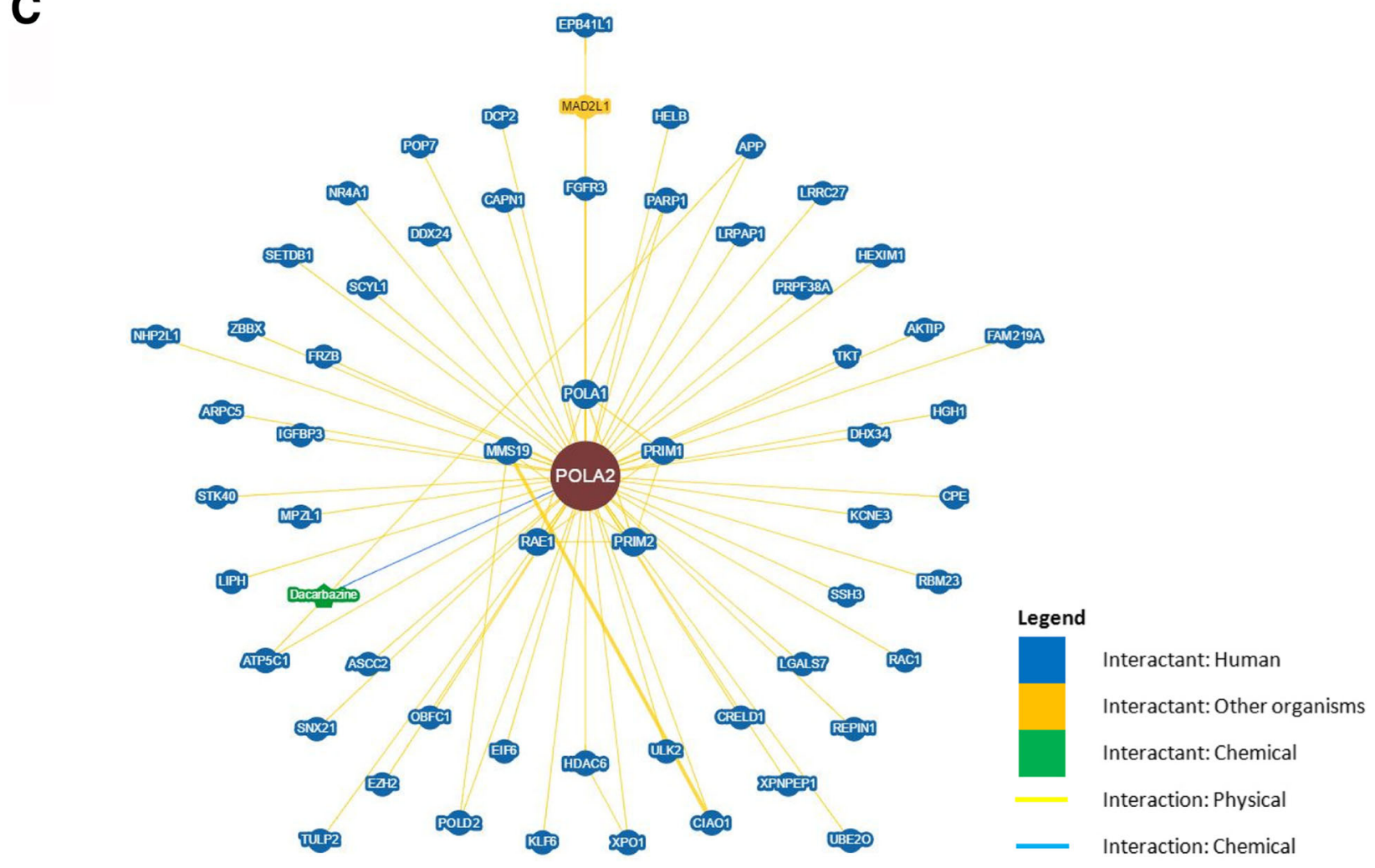

DNA polymerase $\alpha$. primase complex \begin{tabular}{|l|l|l|l|}
\hline a. & $\infty$ & Pri1 & Pn2 \\
\hline
\end{tabular}

DNA polymerase $\delta$ complex \begin{tabular}{|l|c|c|c|}
\hline$\varepsilon 1$ & $\varepsilon 2$ & $\varepsilon 3$ & $\varepsilon 4$ \\
\hline
\end{tabular} MCM cornplex (heliases)
\begin{tabular}{|l|l|}
\hline Mcm2 & Mcm3 \\
\hline Mcra4 & Mcms \\
\hline Mcra6 & Mcm3 \\
\hline
\end{tabular} Clarnp Clarap losder \begin{tabular}{|l|l|l|l|}
\hline PCNA & \multicolumn{3}{|c|}{ Clarmp losder } \\
\hline RFC1 & $\mathrm{RFC2} / 4$ & $\mathrm{RFC} 315$ \\
\hline
\end{tabular}

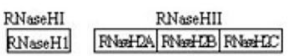
Helicase DNA ligase \begin{tabular}{|l|l|l|l|}
\hline 81 & $\delta 2$ & 83 & 84 \\
\hline
\end{tabular} DNA polymerase $\varepsilon$ corplex

Fig. 1 (See legend on next page.) 
(See figure on previous page.)

Fig. 1 Genomics analysis of POLA2. a Genomic landscape of POLA2 based on the human Dec. 2013 (GRCh38/hg38) assembly. Location of POLA2 is indicated by a red vertical line on chromosome 11. Coding exons are represented by blocks linked by a horizontal line. CpG islands are shown as green blocks. Data associated with integrated transcriptional regulatory elements were retrieved from ENCODE. Comparative genomics analysis using multiple alignments of vertebrate species revealed conservation of the POLA2 gene. Each SNP is shown separately and labeled with the respective SNP ID reported by dbSNP build 146 . Only SNPs that have a minor allele frequency of at least $1 \%$ and are mapped to a single location in the reference genome assembly are shown in the figure. $\mathbf{b}$ Eukaryotic DNA replication complex showing the role of POLA2 (boxed in red) in DNA replication, which involves a complex network of interacting enzymes and proteins. Three DNA polymerases $(a, \delta$ and $\varepsilon$ ) have been identified in eukaryotes. DNA polymerase $a$, including POLA2, forms a complex with DNA primase during the process. Source: Kyoto Encyclopedia of Genes and Genomes (KEGG) pathway database. c Network visualization of POLA2 and its interactants. Physical and genetic interactions, including chemical associations and post-translational modifications, were curated from published datasets and annotated interaction data from NCBI to construct this network

upon knockdown of the POLA2 gene. This implied that other factors and mechanisms might play a role in contributing to gemcitabine resistance and more work needs to be done to explore this avenue.

\section{Discussion}

Previously, we have demonstrated that the POLA2 + 1747 GG/GA SNP is associated with improved survivability and mortality in NSCLC patients [12]. In this current study, we reported that the response to gemcitabine treatment is related to POLA2 and confirmed that the knockdown of POLA2 gene increased the resistance to gemcitabine treatment in human lung cancer cells.

NSCLC is one of the most chemoresistant cancer types. Clinical treatment using traditional chemotherapy agents have reached a plateau in NSCLC patients due to resistance to chemotherapy drugs. Molecular targeted therapy through interruption of the epithelial growth factor receptor was one of the earlier treatment strategies used [13]. However, this strategy failed to achieve remarkable results during clinical trials [14]. Many factors, including genetic heterogeneity, redundant tumor growth as well as survival signaling pathways, likely contributed to the resistance of NSCLC patients to molecular therapies [15]. As such, genetic studies and the understanding of signaling mechanisms may help to identify appropriate treatment strategies for NSCLC patients.

The DNA polymerase $\alpha$ subunit B, which is involved in the initiation of chromosomal DNA replication, is encoded by the POLA2 gene. We have previously reported that different variants of the POLA2 gene may improve the prognosis of NSCLC patients [12]. We have demonstrated that the POLA2 + 1747 GG/GA SNP encodes for a mutant DNA polymerase $\alpha$ subunit B that is predominantly localized in the cytoplasm. This leads to an inhibition in nuclear DNA polymerase $\alpha$ activity, thereby conferring a protective effect on NSCLC patients due to termination of tumor DNA replication. This inhibition of tumor cell proliferation ultimately results in tumor cell death.

Gemcitabine is a potent DNA synthesis inhibitor, which is a deoxycytidine analogue with anti-tumor activity against various solid tumours such as NSCLC, pancreatic cancer, breast cancer and ovarian cancer [16]. Gemcitabine enters the cell through transport by members of the nucleoside transporter family SLC28 and SLC29 $[17,18]$. It is then activated by the deoxycytidine kinase to its monophosphate form in a rate-limiting step. The monophosphate form is then further phosphorylated into gemcitabine triphosphate, which is then incorporated into the DNA by DNA polymerase $\alpha$. Through a process of masked chain termination, DNA synthesis and repair are prematurely halted $[19,20]$. As gemcitabine is involved in the inhibition of DNA synthesis, it is a suitable drug candidate for studying the role of POLA2.

One important aspect of genetic studies is the interactions between SNPs. In a study of SNP-SNP interactions, a small number of SNPs is genotyped and then tested if any interactions are present. Such SNP-SNP interaction studies may help to identify genomic hotspots in human diseases. The possibility of using statistical modeling by many types of regression techniques with straightforward implementation of interaction analysis is also an additional advantage of SNP-SNP interaction studies. We have applied biostatistics to calculate the probability between different SNP pairs and their overall association to the survival of lung cancer patients [12]. From in silico prediction using statistics, we found that POLA2 + 1747 GG/GA SNP together with SLC28A2 + 65 CC SNP were associated with increased median survival. Here, in this study, we validated these statistical findings through wet-lab experiments. We hypothesised that having the POLA + 1747 GG/GA SNP would cause a tumor to be more sensitive to gemcitabine treatment. By performing drug sensitivity tests and sequencing the DNA of human lung cancer cells for the SNP sites of interest, we were able to monitor whether the presence of POLA +1747 GG/GA SNP could affect the response to gemcitabine treatment. Our results showed that all cell lines contained the wild-type POLA2 gene. We then proceeded to silence the POLA2 gene by siRNA transfection and found that instead of causing tumor cell death due to termination of DNA replication, the cell lines became more resistant to gemcitabine treatment. This is the first report to suggest that the POLA2 gene does not act alone but may cooperate with other genes to cause drug resistance to gemcitabine. Indeed, we showed through in silico prediction that several 


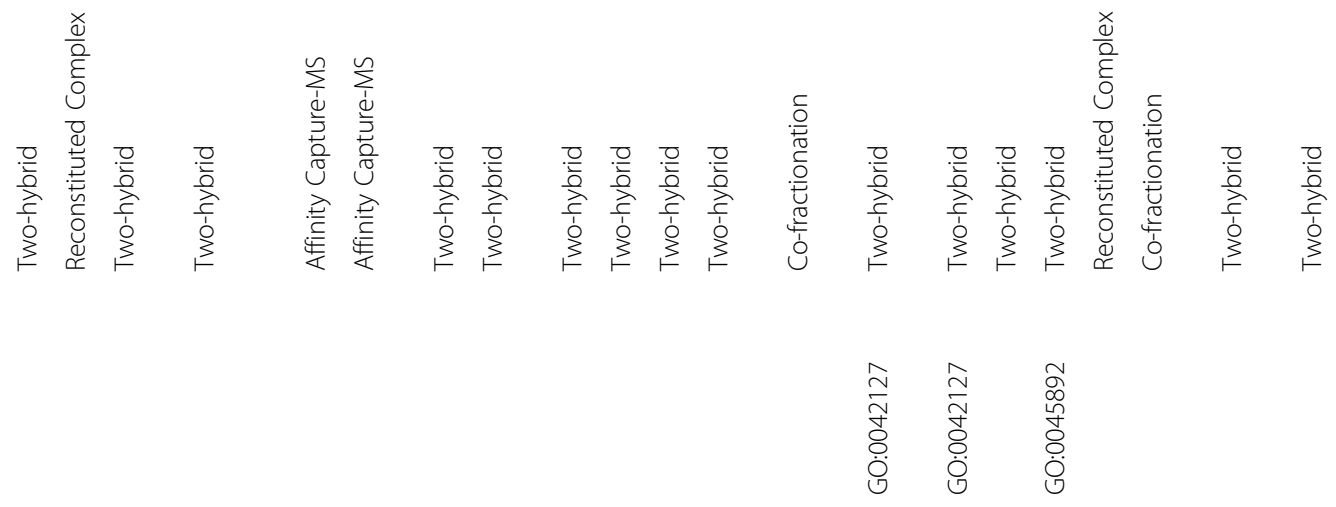

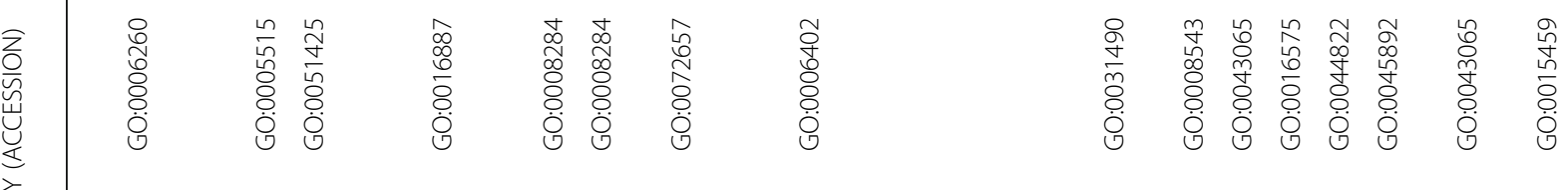

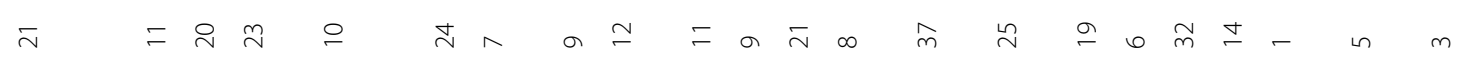

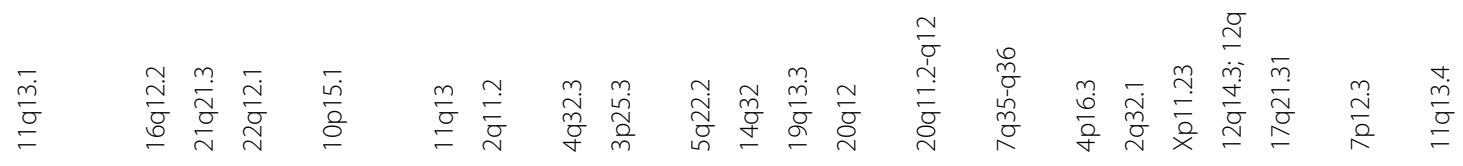

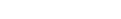

$$
\text { (1) }
$$




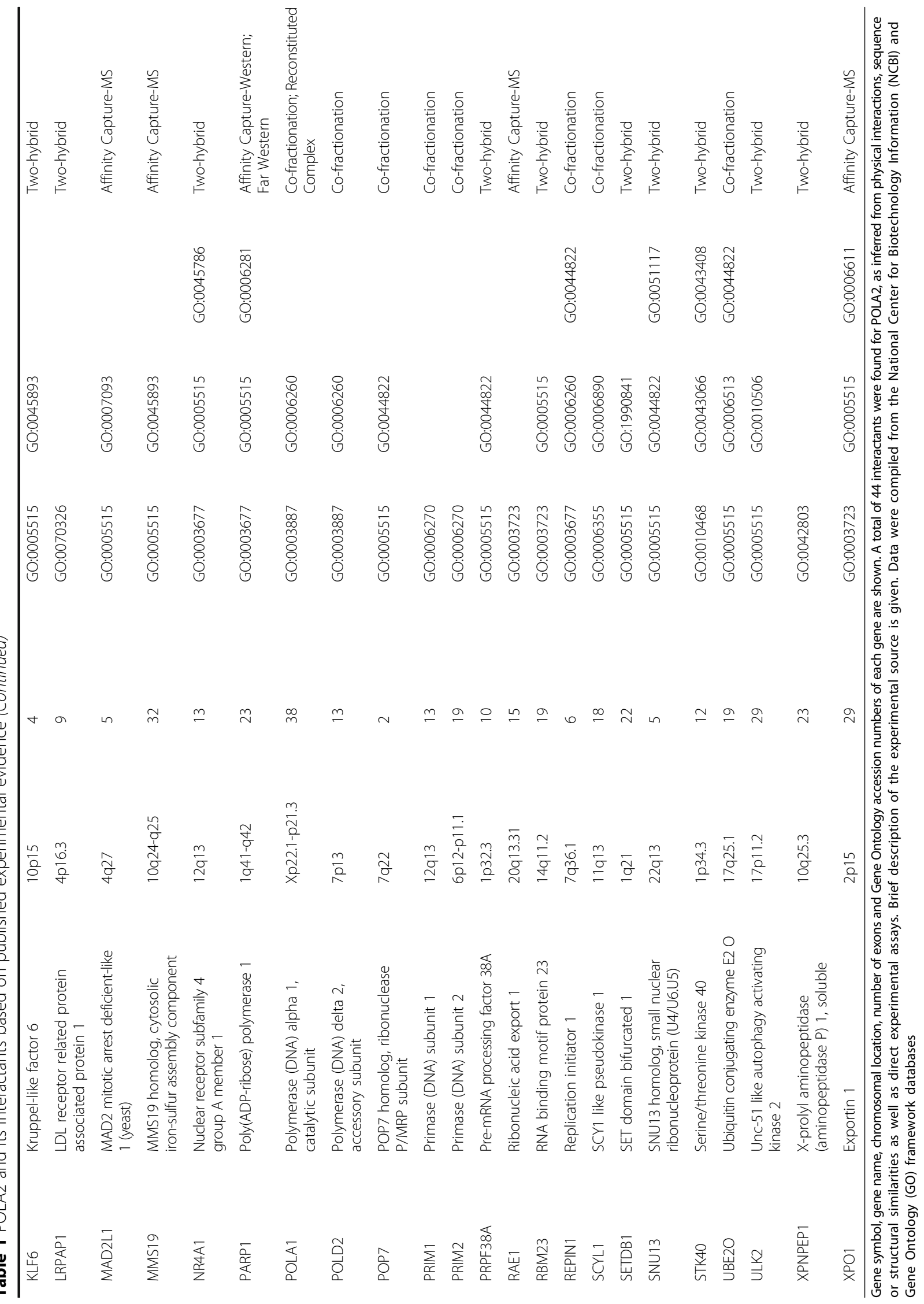




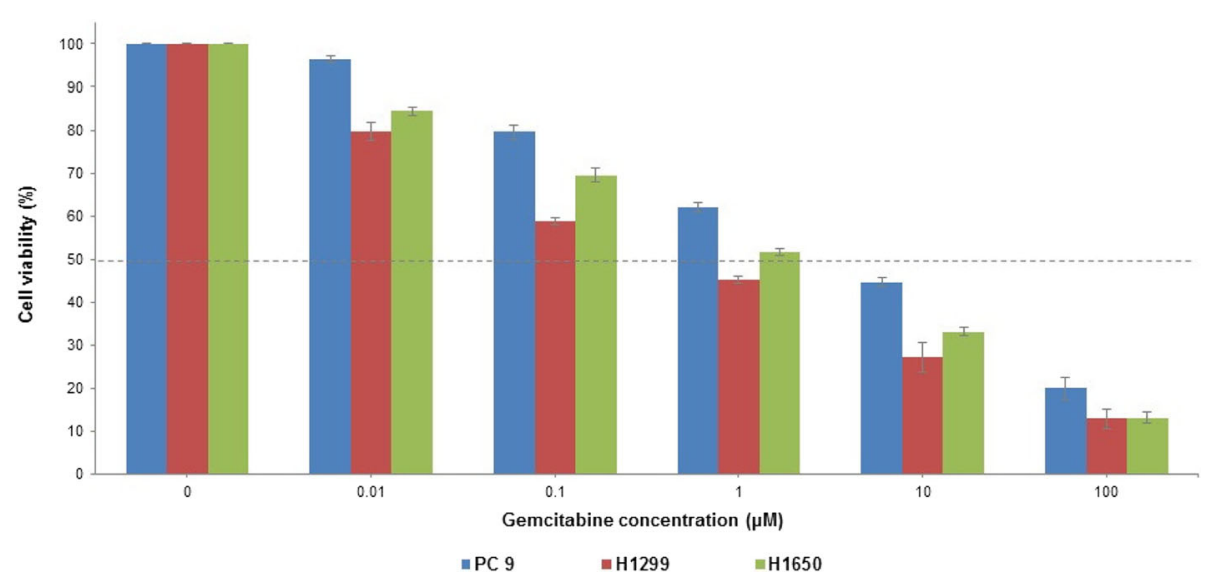

Fig. 2 Effect of gemcitabine on human lung cancer cell lines. Human lung cancer cell lines, namely PC9, H1299 and H1650, were treated with 0.01-100 $\mu \mathrm{M}$ gemcitabine for up to $72 \mathrm{~h}$. Untreated cells ( $0 \mu \mathrm{M}$ gemcitabine) were used as the Control. All experiments were conducted in triplicates and repeated at least 3 times. Data are shown as the mean \pm SD. Dotted line indicates 50\% cell viability

interacting partners of POLA2 exist. Our findings are concomitant with those recently reported by Kang et al. [10], who showed that POLA2 participates in gene fusion events and may act as a prognostic biomarker in gastrointestinal stromal tumors.

Earlier studies have reported other genes that caused resistance to gemcitabine treatment. Davidson et al. [21] proposed that increased expression of the ribonucleotide

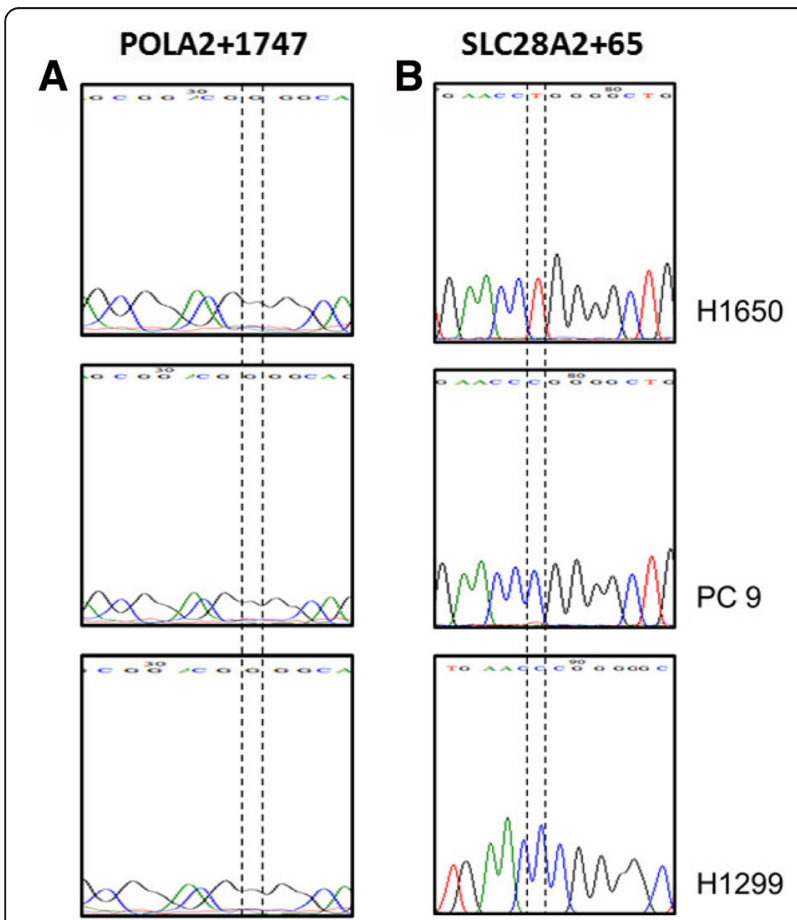

Fig. 3 Detection of SNPs in human lung cancer cell lines. Sequencing plots of H1650, PC9 and H1299 cell lines for (a) POLA2 + 1747 SNP and (b) SLC28A + 65 SNP. All 3 cell lines harboured the wild-type POLA2 + 1747 GG SNP. Only H1650 harboured the mutation SLC28A + 65 CC > CT SNP, while both PC9 and H1299 contained the wild-type SLC28A + 65 CC SNP reductase catalytic subunit M1 (RRM1) gene results in increased resistance to gemcitabine, while Oguri et al. [22] reported that decreased expression of the multidrug resistance protein 5 (MRP5) gene leads to an increase in gemcitabine sensitivity. In addition, studies by Rha et al. [23] showed that patients with the RRM1 haplotypes $2455 \mathrm{~A}>\mathrm{G}$ and $2464 \mathrm{G}>\mathrm{A}$ tend to be genetically more resistant to gemcitabine. Collectively, these studies showed that resistance to gemcitabine are multifactorial, which involves decreased intracellular accumulation and alteration of metabolism [24]. Similar factors include increased gemcitabine degradation enzymes, decreased gemcitabine regulation enzymes and decreased activity of nucleoside transporters (SLC28A1 and SLC29A1) $[20,25,26]$. Other factors such as activation of DNA repair pathways, negative regulation of apoptosis, alterations in cell cycle and cell proliferation pathways, and transition to a more epithelial-to-mesenchymal transition (EMT)-like phenotype can also lead to an increased resistance to gemcitabine treatment.

Our findings showed that POLA2 may be a novel gene that causes resistance to gemcitabine. Similar to the RRM1 gene, POLA2 is involved in DNA synthesis and hence, a decrease in its expression might cause the upregulation of genes that play a role in DNA synthesis and repair. These genes and their polymorphisms should be studied to determine whether there are any SNP-SNP interactions present among them, as well as with the POLA2 gene, that could have led to gemcitabine resistance. Van de Wiel et al. [27] reported chromosomal aberrations as detected by microarray analysis. Genomewide gene expression and SNP microarray analyses may further reveal information on signaling mechanisms and pathways leading to acquired gemcitabine resistance. We are currently exploring this area to study which genes are deregulated, leading to drug 


\section{A}

\section{H1299 cell line}

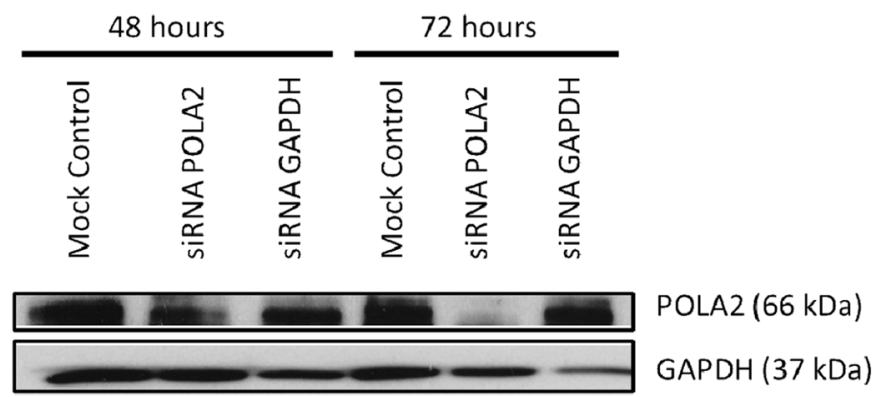

B

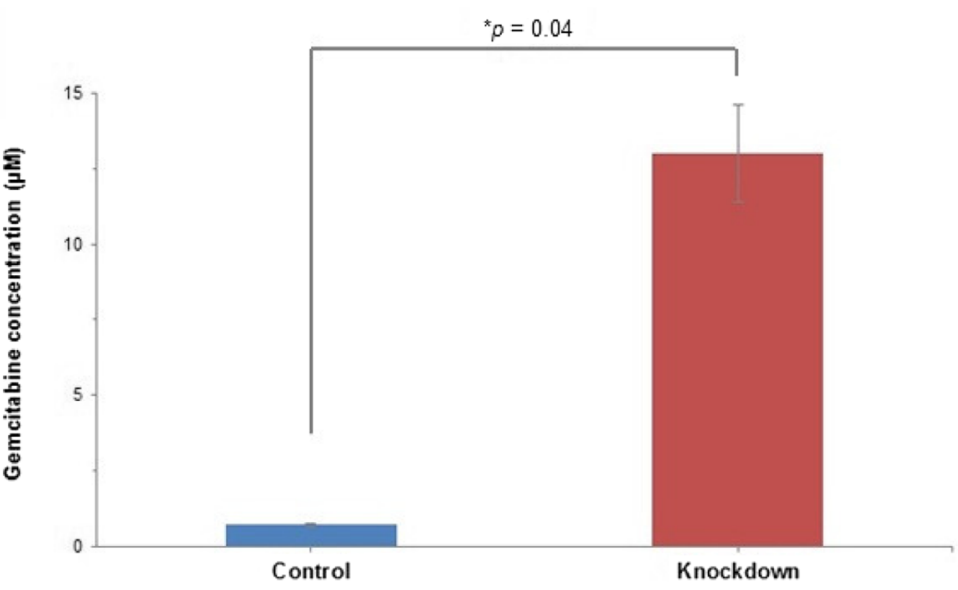

Fig. 4 Effects of POLA2 gene silencing. a Protein expression after knockdown of POLA2 by siRNA. Comparison of POLA2 protein expression in H1299 cell line by Western blotting after 48 and $72 \mathrm{~h}$ of siRNA transfection. Internal control for equal loading: glyceraldeyde phosphate dehydrogenase (GAPDH). $\mathbf{b}$ Chemosensitivity assay by comparing $\mathrm{IC}_{50}$ values before and after knockdown of POLA2. Results indicated that the H1299 cell line became more resistant to gemcitabine treatment after siRNA knockdown of POLA2 (Knockdown), as compared to the non-transfected H1299 cell line having wild-type POLA2 (Control). Experiments were conducted in triplicates and repeated at least 3 times. Data are shown as the $m e a n \pm S D\left({ }^{*} p<0.05\right)$

resistance. Studying the gene expression and polymorphisms of nucleoside transporters and genes involved in DNA synthesis and repair can also help to predict chemosensitivity.

\section{Conclusions}

Taken together, our findings suggested that POLA2 may play a role in gemcitabine resistance. This has important implications as patients with certain variants of the POLA2 gene may be resistant to gemcitabine and may not survive well on gemcitabine treatment. We propose that POLA2 may be used as a prognostic biomarker of patient outcome in NSCLC pathogenesis. More work needs to be done to characterize the correlation between POLA2 and gemcitabine resistance. Functions of the POLA2 + 1747 GG/GA SNP should also be further studied to determine its role in drug resistance and sensitivity. Our earlier report suggested that the presence of SLC28A2 + 65 CC/CT SNP may increase the sensitivity to gemcitabine and thus giving NSCLC patients a better prognosis [12]. However, as shown in Fig. 1 in our current study, SLC28A2 + 65 CC SNP has not been proven to be an interactant of POLA2. Therefore, more studies are needed to investigate whether SLC28A2 + 65 $\mathrm{CC} / \mathrm{CT}$ SNP is associated with a better response to gemcitabine treatment and whether this SLC28A2 $+65 \mathrm{CC} /$ CT SNP indeed interacts with the POLA2 + 1747 GG/ GA SNP. A larger panel of cell lines carrying either or both SNPs needs to be screened to obtain a more comprehensive overview and we are currently working on this aspect.

\section{Methods}

\section{Genomics analysis of POLA2}

The genome sequence of POLA2 was examined using the human Dec. 2013 (GRCh38/hg38) assembly and Encyclopedia of DNA Elements (ENCODE) on the UCSC Genome Browser (https://genome.ucsc.edu/). Human POLA2 sequence was aligned with genome assemblies of other vertebrates and compared using Multiz Alignments Configuration. SNP sites within the POLA2 genomic region were investigated following the Single 
Nucleotide Polymorphism database (dbSNP; build 146). Only SNPs having a minor allele frequency of at least $1 \%$ and mapped to a single location in the human reference genome assembly were flagged and included in subsequent analyses.

\section{Study of SNP-SNP interactions}

SNP-SNP interactions of 21 non-synonymous SNPs in 9 genes involved in gemcitabine transport, metabolism and activity were deduced in silico as reported previously [12]. Briefly, Fisher's exact probability test was used to show that POLA + 1747 GG/GA SNP (rs487989) was the most statistically significant SNP to be associated with mortality. Chi-squared test was employed to assess the association between POLA + 1747 GG/GA SNP and SLC28A2 + 65 CC SNP, which confirmed that this SNP pair gave the best survival outcome [12]. All statistical tests were two-sided and performed using SPSS software version 14.0 (SPSS Inc., Chicago, IL). Differences were considered statistically significant when the $p$-value was less than 0.05 .

\section{Association of POLA2 and other genes}

Gene interactions in association with POLA2 were uncovered using data compiled from the National Center for Biotechnology Information (NCBI) and Gene Ontology (GO) framework databases. Interactants were inferred from physical interactions, sequence or structural similarities, as well as direct experimental assays. The Kyoto Encyclopedia of Genes and Genomes (KEGG) pathway database was used to further mine for major pathways involving POLA2. Only experimentally validated interactants were chosen to generate a network of interactions using the Biological General Repository for Interaction Datasets (BioGRID), in which POLA2 plays a central role.

\section{Cell culture}

Human lung cancer H1650 (NCI-H1650) and H1299 (NCI-H1299) cell lines were purchased from American Type Culture Collection (ATCC; Rockville, MD). Human lung adenocarcinoma PC-9 cell line was purchased from RIKEN BioResource Center (Ibaraki, Japan). All cell lines were cultured in Roswell Park Memorial Institute (RPMI) 1640 medium (Gibco; Thermo Fisher Scientific, Waltham, MA) containing $10 \%$ fetal bovine serum (HyClone; GE Healthcare Life Sciences, Marlborough, MA) as recommended by the respective suppliers.

\section{Drug treatment}

Cells were seeded into 96-well plates (Nunc; Thermo Fisher Scientific) at a density of $2 \times 10^{4}$ cells per well in $100 \mu \mathrm{l}$ culture medium and incubated at $37^{\circ} \mathrm{C}$ overnight. Cells were treated with various concentrations of gemcitabine (Sigma-Aldrich, St. Louis, MI) on the following day to determine the $\mathrm{IC}_{50}$ values. Final concentrations of gemcitabine treatment were 0.01, 0.1, 1,10 and $100 \mu \mathrm{M}$. Cell viability was determined using the CellTiter 96 AQueous One Solution Cell Proliferation Assay (MTS; Promega, Madison, WI) following manufacturer's instructions.

\section{Sequencing}

Cell lines were sequenced to confirm the presence of the POLA2 + 1747 and SLC28A2 + 65 SNPs (Axil Scientific, Singapore). Sequencing results were then correlated with the $\mathrm{IC}_{50}$ values to assess the SNP association previously reported [12].

\section{Silencing of POLA2 +1747 by siRNA transfection}

Silencing of the POLA2 gene was done by siRNA transfection using DharmaFECT transfection reagents (Dharmacon, Lafayette, CO) following manufacturer's instructions. The H1299 cell line was used, as this cell line had been tested and validated by the manufacturer. Transfection durations were 24, 48 and $72 \mathrm{~h}$. Western blotting was then performed to check for protein expression of the silenced gene, as described previously [12].

\section{Declarations}

This article has been published as part of BMC Genomics Volume 17 Supplement 3, 2016: 15th International Conference On Bioinformatics (INCOB 2016). The full contents of the supplement are available online at https:// bmcgenet.biomedcentral.com/articles/supplements/volume-17-supplement-3

\section{Funding}

Publication of this article was funded by the Agency for Science, Technology and Research (A*STAR) Biomedical Research Council (BMRC) Strategic

Positioning Fund (SPF; SPF2012/002) and Joint Council Office (JCO) Grant (JCOAG04_FG03_2009).

Availability of data and materials

Available upon request.

Authors' contributions

TLM conceptualized the project. TLM, WPY and VK designed the study. VK performed in silico analyses. HYK and WLT conducted the experiments. VK, HYK and WLT interpreted and analyzed the experimental data. VK and HYK wrote the manuscript. All authors read and approved the final manuscript.

Competing interests

The authors declare that they have no competing interests.

Consent for publication

Not applicable.

Ethics approval and consent to participate

Not applicable.

\section{Author details}

${ }^{1}$ National University Cancer Institute Singapore, National University Health System, Singapore, Singapore. ${ }^{2}$ Cancer Science Institute of Singapore, National University of Singapore, Singapore, Singapore. ${ }^{3}$ Institute for Infocomm Research, Agency for Science, Technology and Research, Singapore, Singapore.

Published: 22 December 2016 


\section{References}

1. Molina JR, Yang P, Cassivi SD, Schild SE, Adjei AA. Non-small cell lung cancer: epidemiology, risk factors, treatment, and survivorship. Mayo Clin Proc. 2008:83:584-94.

2. Gadgeel SM, Ramalingam SS, Kalemkerian GP. Treatment of lung cancer Radiol Clin North Am. 2012:50:961-74.

3. Toschi L, Finocchiaro G, Bartolini S, Gioia V, Cappuzzo F. Role of gemcitabine in cancer therapy. Future Oncol. 2005:1:7-17.

4. Alvarellos ML, Lamba J, Sangkuhl K, Thorn CF, Wang L, Klein DJ, Altman RB, Klein TE. PharmGKB summary: Gemcitabine Pathway. Pharmacogenet Genomics. 2014:24:564-74.

5. Erčulj N, Kovač V, Hmeljak J, Franko A, Dodič-Fikfak M, Dolžan V. The influence of gemcitabine pathway polymorphisms on treatment outcome in patients with malignant mesothelioma. Pharmacogenet Genomics. 2012;22:58-68.

6. Li L, Schaid DJ, Fridley BL, Kalari KR, Jenkins GD, Abo RP, Batzler A, Moon I, Pelleymounter L, Eckloff BW, Wieben ED, Sun Z, Yang P, Wang L. Gemcitabine metabolic pathway genetic polymorphisms and response in patients with non-small cell lung cancer. Pharmacogenet Genomics. 2012;22:105-16.

7. Lehman IR, Kaguni LS. DNA polymerase alpha. J Biol Chem. 1989;264:4265-8.

8. Yamtich J, Sweasy JB. DNA polymerase family X: function, structure, and cellular roles. Biochim Biophys Acta. 2010;1804:1136-50.

9. Collins KL, Russo AA, Tseng BY, Kelly TJ. The role of the $70 \mathrm{kDa}$ subunit of human DNA polymerase alpha in DNA replication. EMBO J. 1993;12:4555-66.

10. Kang G, Yun H, Sun CH, Park I, Lee S, Kwon J, Do I, Hong ME, Vrancken MV, Lee J, Park JO, Cho J, Kim KM, Sohn TS. Integrated genomic analyses identify frequent gene fusion events and VHL inactivation in gastrointestinal stromal tumors. Oncotarget. 2016;7:6538-51.

11. Willis S, Villalobos VM, Gevaert O, Abramovitz M, Williams C, Sikic BI, Leyland-Jones B. Single gene prognostic biomarkers in ovarian cancer: A meta-analysis. PLoS One. 2016;11:e0149183.

12. Mah TL, Yap XN, Limviphuvadh V, Li N, Sridharan S, Kuralmani V, Feng M, Liem N, Adhikari S, Yong WP, Soo RA, Maurer-Stroh S, Eisenhaber F, Tong JC. Novel SNP improves differential survivability and mortality in non-small cell lung cancer patients. BMC Genomics. 2014;15 Suppl 9:S20.

13. Shepherd FA. Angiogenesis inhibitors in the treatment of lung cancer. Lung Cancer. 2001;34:S81-89.

14. Gatzemeier U, Groth G, Butts C, Van Zandwijk N, Shepherd F, Ardizzoni A Barton C, Ghahramani P, Hirsh V. Randomized phase II trial of gemcitabinecisplatin with or without trastuzumab in HER2-positive non-small-cell lung cancer. Ann Oncol. 2004:15:19-27.

15. Denlinger CE, Rundall BK, Keller MD, Jones DR. Proteasome inhibition sensitizes non-small-cell lung cancer to gemcitabine-induced apoptosis. Ann Thorac Surg. 2004;78:1207-14.

16. Woo HI, Kim KK, Choi H, Kim S, Jang KT, Yi JH, Park YS, Park JO, Lee SY Effect of genetic polymorphisms on therapeutic response and clinical outcomes in pancreatic cancer patients treated with gemcitabine. Pharmacogenomics. 2012;13:1023-35.

17. Mackey JR, Yao SY, Smith KM, Karpinski E, Baldwin SA, Cass CE, Young JD. Gemcitabine transport in xenopus oocytes expressing recombinant plasma membrane mammalian nucleoside transporters. J Natl Cancer Inst. 1999;91:1876-81.

18. Kong W, Engel K, Wang J. Mammalian nucleoside transporters. Curr Drug Metab. 2004;5:63-84

19. Heinemann V, Hertel LW, Grindey GB, Plunkett W. Comparison of the cellular pharmacokinetics and toxicity of 2',2'-difluorodeoxycytidine and 1-beta-D-arabinofuranosylcytosine. Cancer Res. 1988;48:4024-31.

20. Plunkett W, Huang P, Xu YZ, Heinemann V, Grunewald R, Gandhi V. Gemcitabine: metabolism, mechanisms of action, and self-potentiation. Semin Oncol. 1995;22:3-10

21. Davidson JD, Ma L, Flagella M, Geeganage S, Gelbert LM, Slapak CA. An increase in the expression of ribonucleotide reductase large subunit 1 is associated with gemcitabine resistance in non-small cell lung cancer cell lines. Cancer Res, 2004:64:3761-6.

22. Oguri T, Achiwa H, Sato S, Bessho Y, Takano Y, Miyazaki M, Muramatsu H, Maeda H, Niimi T, Ueda R. The determinants of sensitivity and acquired resistance to gemcitabine differ in non-small cell lung cancer: a role of ABCC5 in gemcitabine sensitivity. Mol Cancer Ther. 2006;5:1800-6.

23. Rha SY, Jeung HC, Choi YH, Yang WI, Yoo JH, Kim BS, Roh JK, Chung HC. An association between RRM1 haplotype and gemcitabine-induced neutropenia in breast cancer patients. Oncologist. 2007;12:622-30.
24. Bergman AM, Pinedo HM, Peters GJ. Determinants of resistance to 2',2'difluorodeoxycytidine (gemcitabine). Drug Resist Update. 2002;5:19-33.

25. Bergman AM, Giaccone G, van Moorsel CJ, Mauritz R, Noordhuis P, Pinedo HM, Peters GJ. Cross-resistance in the 2',2'-difluorodeoxycytidine (gemcitabine)-resistant human ovarian cancer cell line AG6000 to standard and investigational drugs. Eur J Cancer. 2000;36:1974-83.

26. Fukunaga AK, Marsh S, Murry DJ, Hurley TD, McLeod HL. Identification and analysis of single-nucleotide polymorphisms in the gemcitabine pharmacologic pathway. Pharmacogenomics J. 2004;4:307-14.

27. Van de Wiel MA, Costa JL, Smid K, Oudejans CB, Bergman AM, Meijer GA, Peters GJ, Ylstra B. Expression microarray analysis and oligo array comparative genomic hybridization of acquired gemcitabine resistance in mouse colon reveals selection for chromosomal aberrations. Cancer Res. 2005;65:10208-13.

\section{Submit your next manuscript to BioMed Central and we will help you at every step:}

- We accept pre-submission inquiries

- Our selector tool helps you to find the most relevant journal

- We provide round the clock customer support

- Convenient online submission

- Thorough peer review

- Inclusion in PubMed and all major indexing services

- Maximum visibility for your research

Submit your manuscript at www.biomedcentral.com/submit
C) Biomed Central 